EPJ Web of Conferences 45, 01044 (2013)

DOI: $10.1051 /$ epjconf/20134501044

(C) Owned by the authors, published by EDP Sciences, 2013

\title{
Investigation of different wall profiles on energy consumption and baking time in domestic ovens
}

\author{
O. Isik ${ }^{1}$, O. Temel ${ }^{1}$, A. Celik ${ }^{1}$, T. Sari ${ }^{1}$ and S. U. Onbasioglu ${ }^{1}$ \\ ${ }^{1}$ Istanbul Technical University, Mechanical Engineering, 34437 Istanbul, Turkey
}

\begin{abstract}
This present study, has aimed to examine and improve the momentum and heat transport mechanism in a domestic oven. At the beginning, the experimental study has been carried out in the oven that analyzing heat transfer behavior of the oven. During the preparation and procedure of the energy consumption experiments, standards determined in EN 50304 had been used. In addition to experimental results, the numerical simulation has showed that increasing diffusion over the walls and advection in the center, the performance of the oven can be improved. Using this idea, two different models have been set up in the oven walls and have been experimentally and numerically studied. Finally, the results have been compared with the original case that validates the initial idea by improving the performance of the oven.
\end{abstract}

\section{Introduction}

As it is noticed that the energy resources of our planet is alarming, the researches have started to give their attention to decrease the consumptions. For that purpose, the household appliances are one of the important fields to focus. Thanks to these studies, the efficiency levels are improving. During 90's the energy efficiency of a domestic oven was in approximately $12-13 \%$, nowadays the levels are lift up to $25 \%$ [1]. Nevertheless, the standardization of the energy consumption experiments has become to be identified more detailed by the comities. European Committee for Electrotechnical Standardization (CELENEC) has announced in 1996 and, gave its final version in 2005 as EN 50304 for the energy consumption experiments for domestic ovens [2].

\subsection{Experimental procedure}

The experiments are carried according to standard EN 50304 and on a built-in oven shown in figure 1 .

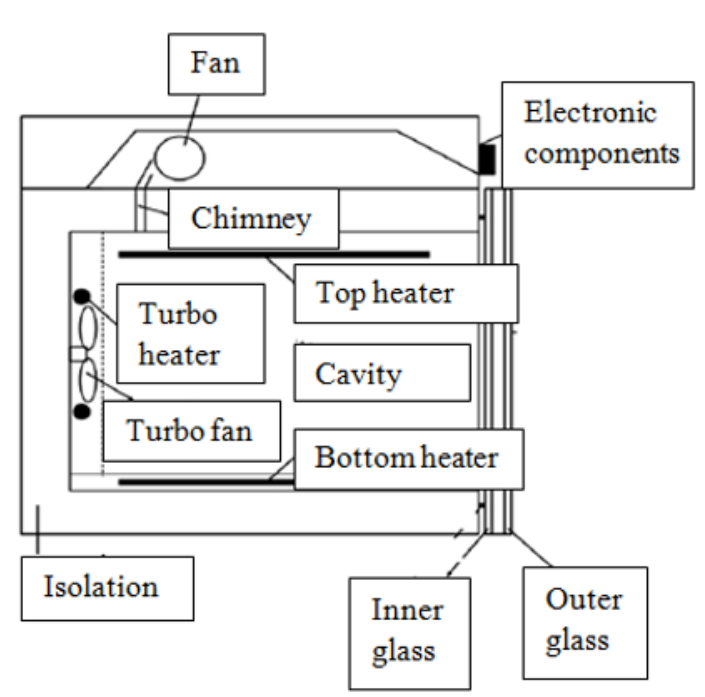

Fig. 1. Built-in oven used in the study

In the standards, a porous brick is being used as resembling the food. EN 50304 standard determines the dimensions and physical properties. The dimension of the brick is shown in figure 2. 
Two thermocouples measuring the center temperature of the brick

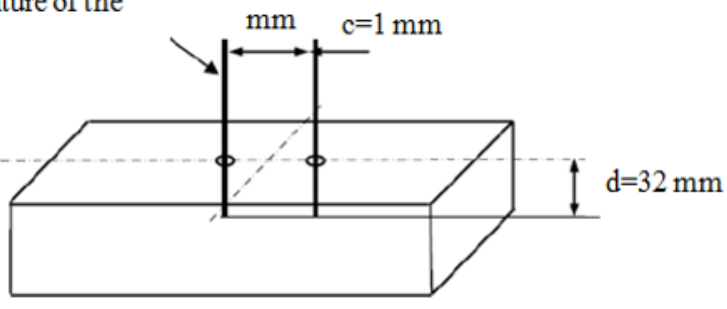

Fig. 2. Dimensions of standard porous brick

The physical properties of the standart brick is tabulated in table 1 .

Table 1. Physical properties of the standard porous brick

\begin{tabular}{|c|c|}
\hline Brick name & Hipor $450[3]$ \\
\hline Dry density $\left(\mathrm{kg} / \mathrm{m}^{3}\right)$ & $550 \pm 40$ \\
\hline Total porosity $(\%)$ & 77 \\
\hline Dry weight $(\mathrm{g})$ & $920 \pm 75$ \\
\hline Absorption of water mass $(\mathrm{g})$ & $1050 \pm 50$ \\
\hline
\end{tabular}

The porous brick is pretreated according to 8.3.3.1 item, with forced air circulation at $\geq 175^{\circ} \mathrm{C}$. After it is dried in the oven, the dry weight is measured. The hot brick left to be cooled down to room temperature and it is put into a water container in a refrigerator set to $5 \pm 2{ }^{\circ} \mathrm{C}$. The room temperature should be set for $23 \pm 2{ }^{\circ} \mathrm{C}$ by an air conditioner during the experiment.

After the brick is prepared, it should be located in the center of the oven cavity. When the both thermocouples measured the center of the brick rises $55 \mathrm{~K}$, the brick is taken from cavity and weight once more. The measured energy consumption and baking time at that moment are noted.

After the brick is taken from the cavity, the center of the cavity temperature should be measured for 5 cycles or at least for 1 hour. The average of the temperature is noted as the center temperature of the oven.

There are two different modes and three different temperature set. If the turbo fan is open it is called 'turbo mode', if only upper and bottom heaters are on, it is called 'static mode'. The temperature sets are shown in table 2.

Table 2. Center cavity temperatures

\begin{tabular}{|c|c|c|c|}
\hline Mode & \multicolumn{3}{|c|}{ Temperature } \\
\hline Static mode & $140 \mathrm{~K}$ & $180 \mathrm{~K}$ & $220 \mathrm{~K}$ \\
\hline Turbo mode & $135 \mathrm{~K}$ & $155 \mathrm{~K}$ & $175 \mathrm{~K}$ \\
\hline
\end{tabular}

\subsection{Experimental setup}

According to EN 50304 standard the listed quantities are being measured during the experiment. The measured quantities should be in the limitations given in the table 3 .

Table 3. Tolerances of the measured quantities

\begin{tabular}{|c|c|c|}
\hline Quantity & Unit & Tolerance \\
\hline Voltage & $\mathrm{V}$ & $230 \pm 1 \%$ \\
\hline Current & $\mathrm{A}$ & $\pm 1 \%$ \\
\hline Frequency & $\mathrm{Hz}$ & $\pm 1 \%$ \\
\hline Temperature & ${ }^{\circ} \mathrm{C} / \mathrm{K}$ & $\pm 1{ }^{\circ} \mathrm{C} / \mathrm{K}$ \\
\hline Time & $\mathrm{s}$ & $\pm 5 \mathrm{~s}$ \\
\hline Brick weight & $\mathrm{g}$ & $\pm 3 \mathrm{~g}$ \\
\hline Energy & $\mathrm{Wh}$ & \pm 1.5 or $\pm 10 \mathrm{Wh}$ \\
\hline
\end{tabular}

The standard determines both temperature and energy measurement during the experiment. The schematic view of the experimental setup is shown in figure 3 .

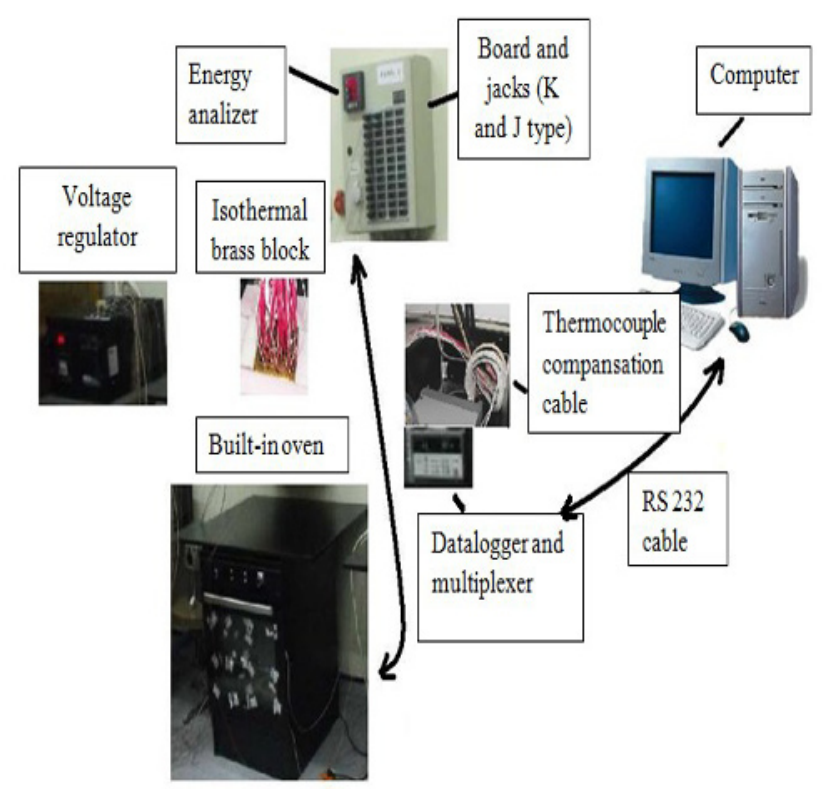

Fig. 3. Schematic view of the experimental setup

In the experimental setup, temperature is measured by both $\mathrm{K}$ and $\mathrm{J}$ type thermocouples. Female jacks and energy analyzer are assembled in the data gathering board. There are thermocouple compensation cables providing the connection between the jacks and both datalogger and isothermal brass block. While the block ensures the calibration of the temperature measurement, datalogger collects the data and deliver it to a computer by a RS 232 cable. The voltage regulator keeps the system in $230 \pm 1 \%$ $\mathrm{V}$ tolerance. 


\subsection{Energy budget study}

As an initial study, the 'energy budget' of the built-in oven has been examined in the research. 'Energy budget' mean to be analyze the items of the total energy consumed. By this way, the aspect of the study to improve the performance of the oven could be much clear. The total energy consumption is measured by the experimental set up. Moreover, the energy consumption items can be categorized such:

- Heat storage of brick

- Heat storage of components

- Heat loses such:

By this way, efficiency of the system can be identified

$$
\text { Efficiency }=\frac{\text { Heat storage of brick }}{\text { Total energy consumption of the oven }}
$$

The experiments have been done for three different temperature set; $140 \mathrm{~K}, 180 \mathrm{~K}$ and $220 \mathrm{~K}$. The total energy consumption of the oven is divided into two parts. The first part, called 'preheating' while the upper and bottom heaters are always on whereas, in the 'cycle' part, the heaters are corresponding to an on-off cycle algorithm. For $180 \mathrm{~K}$, the results of the initial study are given in figure 4, 5 and 6 [4].

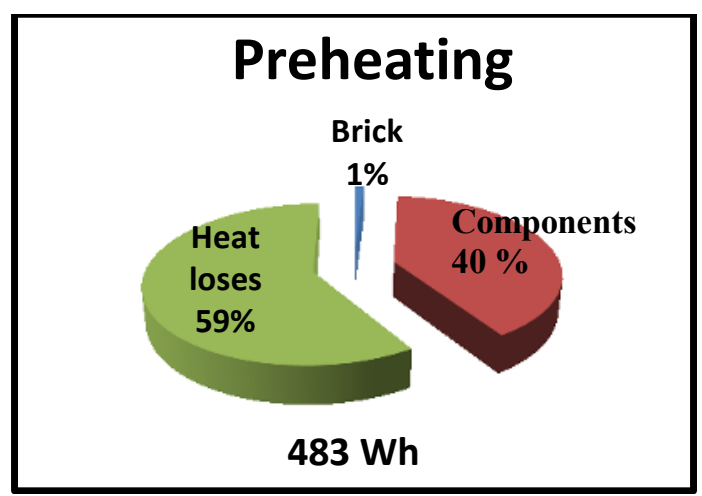

Fig. 4. Energy budget study; $180 \mathrm{~K}$ temperature set -preheating

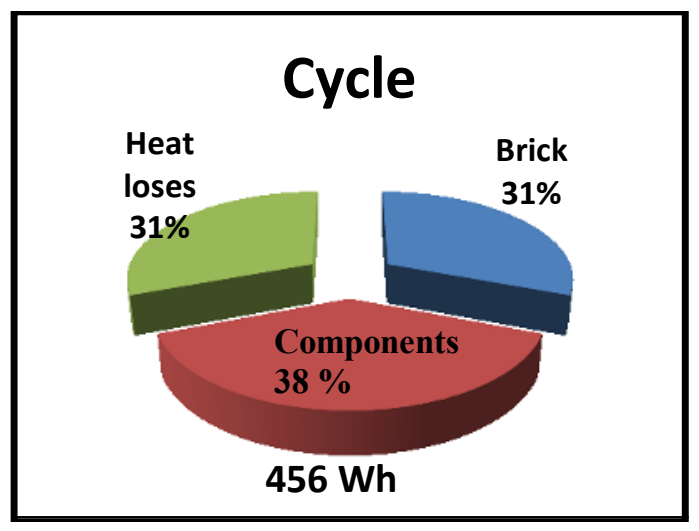

Fig. 5. Energy budget study; $180 \mathrm{~K}$ temperature set -cycle

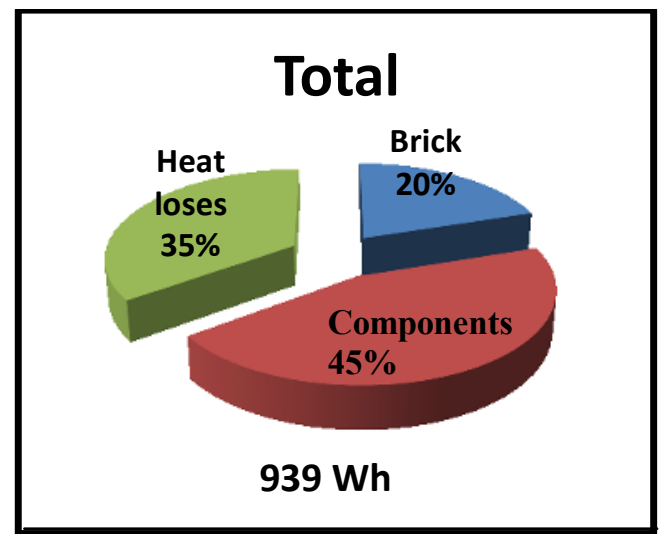

Fig. 6. Energy budget study; $180 \mathrm{~K}$ temperature set -total

As it can be seen from the figure, during preheating section, most of the energy is consumed in order to heat the components of the oven. Although, nearly half of the energy is consumed in this part, only $1 \%$ of the energy is absorbed by the brick. However, in the cycle part, the heat fluxes are becoming much homogenous and the heat is directed to the center of the oven.

From an initial point of view it can be said that, in order to decrease energy consumption:

- The heat loses and heat stored by the components can be decreased in order to decrease the energy consumed in preheating section. (E.g. decreasing the heat capacity of the components or using a different type of insulation)

- During the cycle section, if it is possible to improve heat flux in the center of the cavity the brick can store much energy. (Increasing diffusion over the walls and advection in the center, the performance of the oven can be improved)

\section{Theoretical analysis and previous studies}

\subsection{The rough wall option}

As well-established by the theory of fluid dynamics, the rate of heat transfer on a fluid-solid interface is mathematically expressed by the temperature gradient on the surface. The main aim of this present study and the previous ones is to decrease the heat transfer from cavity to insulation due to energy efficiency concerns. As the rate of energy consumption for the baking process instead of heating of components and insulation, shorter baking time would be achieved. The temperature gradients on cavity surfaces would become less shaper if the boundary layer thicknesses on these surfaces would increase. The boundary layer thickness is proportional to frictional forces which could be increased by any kind of roughness elements. When the boundary layer is thicker, lower velocity values would be observed on the surface of cavity walls and this would provide less sharp temperature gradients. In another way of expressing, when the scale of frictional forces 
adjacent to cavity walls increases, a diffusion-dominated heat and momentum transport would be provided close to the cavity walls. Previous numerical studies on domestic built-in ovens have shown that, contrary to the desired transport mechanism, advection-dominated flow regime appear close to walls instead of center of cavity where the baking is being conducted.

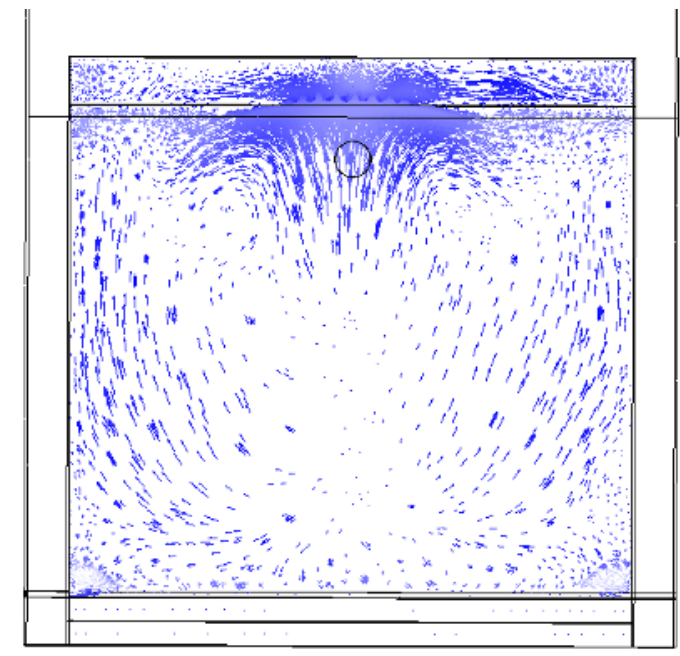

Fig. 7. Velocity vectors - plane perpendicular to gravitational direction

\subsection{The wavy wall option}

As indicated before, energy consumption might be reduced by providing low velocity values on the surface of cavity walls. On the framework of this idea, it is predicted that implementing wavy wall profiles on the cavity walls, would expose flow regions with re-circulations and very low velocity values so called as dead-flow regions. Similar to the rough wall case, advection-dominated heat and momentum transport would be observed at centre of cavity. It must be also noted that, in addition to this positive effect on energy consumption, implementation of wavy walls may also reduce the energy efficiency of oven as the heat transfer area would be increased. On contrary to the statement claiming that dead-flow regions may provide diffusion-based flow regime, it is possible to observe micro-scale turbulent wakes, not also in temporal scale but also in spatial scale. These turbulent wakes may increase the rate of transport close to cavity walls and result in the increase of heat losses from cavity. In other words, implementation of wavy walls on to the cavity walls is a matter of engineering optimization case, which must be carried out with also the aspects of radiative heat transfer. By using approximate boundary conditions a CFD analysis has been conducted for the wavy wall option to examine the flow regime.

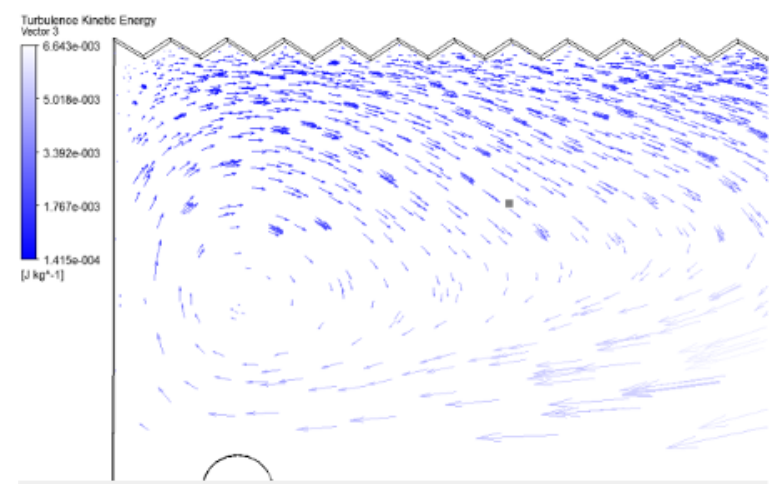

Fig. 8. Velocity vectors - plane perpendicular to gravitational direction

CFD results which are based on approximate boundary conditions have shown that advection-dominated flow regime occured at the centre of cavity and there is no noticeble turbulence kinetic energy peaks adjacent to walls. For the case of radiative heat transfer, as the wavy wall reflects and scatters to itself, a spatially more homogeneous temperature distribution is to be expected. Previous numerical and experimental studies on the present domestic built-in ovens, have shown that both negative and positive Nusselt Number values are obtained for the side walls of cavity. In other words, previous studies have shown that side walls of cavity are not only heat sinks but also heat sources for the fluid inside the cavity.

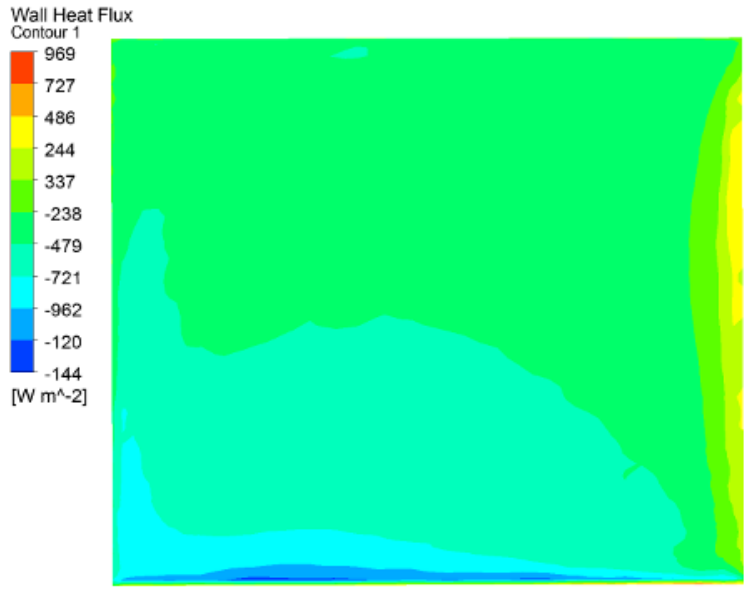

Fig. 9. Total Wall Fluxes on Side Wall

Consequently, it is likely to claim that it is possible to provide a positive uniform-like wall heat flux, by constituting quasi-homogeneous temperature distribution on the side-wall of cavity. Implementation of wavy walls is expected to achieve this goal by the self-scattering and selfreflecting facts. 


\section{Experimental studies}

By the initial aspect summurized above, two different models have been set up in the cavity and studied experimentally. In the previous studies, the research team has been studied to put different reflective geometries inside the cavity walls [5]. In this study, it has been shown that the effective model improved not only radiation but also convection. By improving the heat transfer coefficient among the side walls of the oven, the heat transfer performance of the oven can be raised. In the light of this study, research team have been developed two different models to place in the cavity walls.

\subsection{Experimental results}

After placing the models inside the cavity, standard energy consumption experiments are being carried. Not only energy consumption and baking time, but also heat transfer coefficient over the side walls heat been measured and compared with the original case. These comparisons have been tabulated in table 4 .

Table 4. Energy consumption and baking time comparison with original case

\begin{tabular}{|c|c|c|c|c|c|c|}
\hline \multirow{2}{*}{} & \multicolumn{2}{|c|}{140 K } & \multicolumn{2}{c|}{180 K } & \multicolumn{2}{c|}{220 K } \\
\cline { 2 - 7 } & $\begin{array}{c}\text { Energy } \\
\text { cons. } \\
\text { (Wh) }\end{array}$ & $\begin{array}{c}\text { Baking } \\
\text { time } \\
\text { (min) }\end{array}$ & $\begin{array}{c}\text { Energy } \\
\text { cons. } \\
\text { (Wh) }\end{array}$ & $\begin{array}{c}\text { Baking } \\
\text { time } \\
\text { (min) }\end{array}$ & $\begin{array}{c}\text { Energy } \\
\text { cons. } \\
\text { (Wh) }\end{array}$ & $\begin{array}{c}\text { Baking } \\
\text { time } \\
\text { (min) }\end{array}$ \\
\hline $\begin{array}{c}\text { Original } \\
\text { case }\end{array}$ & 835 & 50.9 & 947 & 41.8 & 1064 & 37.0 \\
\hline $\begin{array}{c}\text { Wavy } \\
\text { wall }\end{array}$ & 808 & 47.1 & 917 & 40.7 & 1047 & 36.6 \\
\hline $\begin{array}{c}\text { Rough } \\
\text { wall }\end{array}$ & 779 & 46.2 & 939 & 39.9 & 1043 & 36.8 \\
\hline
\end{tabular}

As summarized in table 4, the most efficient models are obtained for $140 \mathrm{~K}$ and $220 \mathrm{~K}$ with rough wall design, for $180 \mathrm{~K}$ with wavy wall design. These models are provided $6.7 \%, 3.3 \%$ and $2 \%$ energy consumption and $9.2 \%, 2.6$ $\%$ and $0.5 \%$ less baking time respectively.

Moreover, in order to examine the model's contribution to convective heat transfer among the walls convective heat transfer coefficient has been compared in figure 10 and 11 .

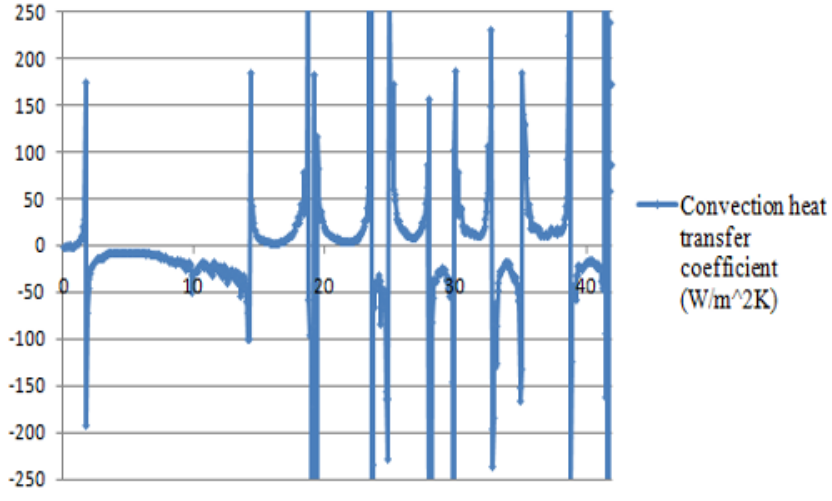

Fig. 10. Heat transfer coefficient over the side wall - original case

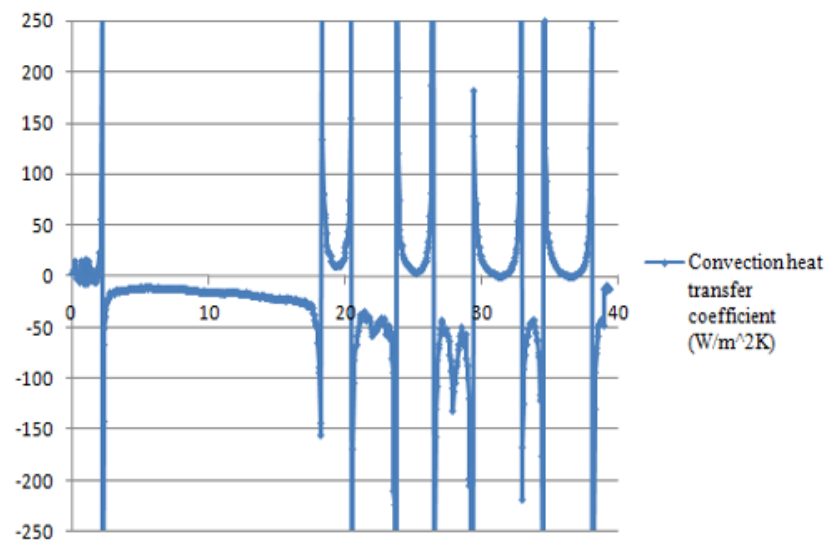

Fig. 11. Heat transfer coefficient over the side wall - wavy wall

\section{Computational fluid dynamics study on the remarkable convective heat transfer coefficient peaks}

Experimentally, convective heat transfer coefficients have been determined. Results have shown that during the not only in cycle-region but also in pre-heating period, remarkable convective heat transfer coefficient peaks have been observed. Pre-heating period is the linear-like portion of transient temperature distribution and the referred cycle region, and the time period taken into account for numerical solution are shown in figure 12; 


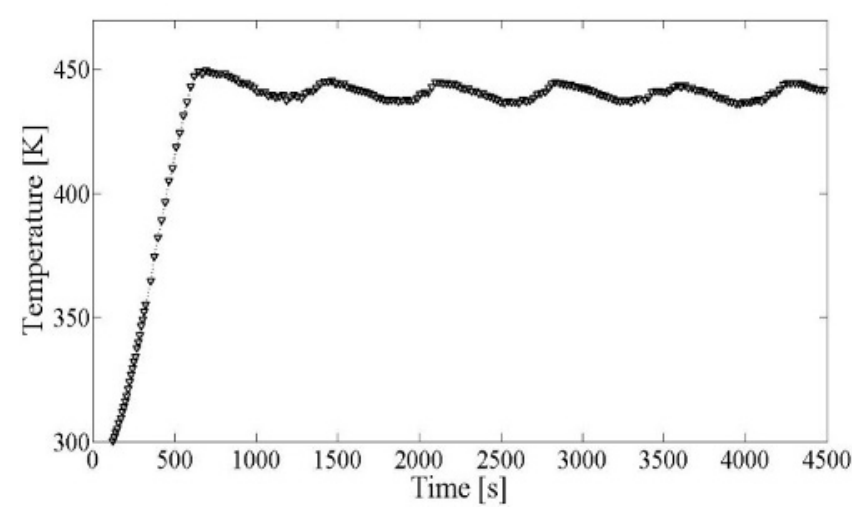

Fig. 12. Transient temperature distribution at the centre of cavity

As can be seen from the given figure 13, these convective heat transfer coefficient peaks may seem to be stochastically and it is possible to discard those measurement data under the consideration of experimental error.

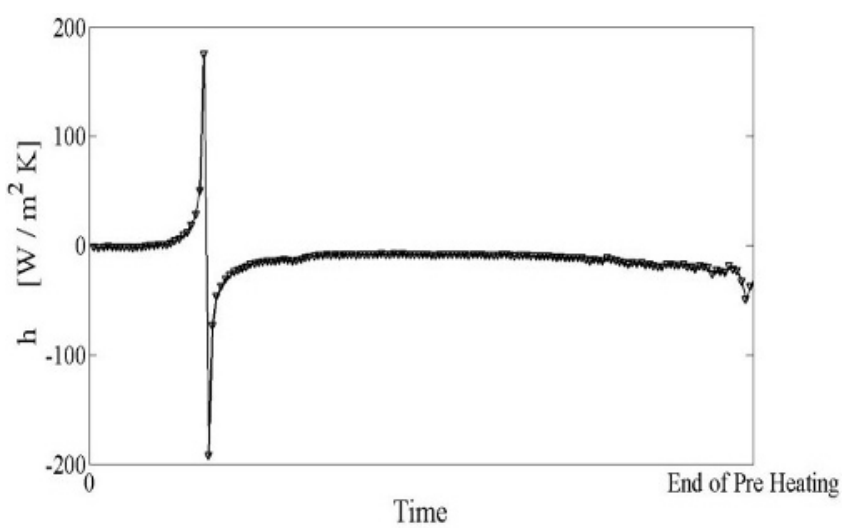

Fig. 13. Transient convective heat transfer coefficient distribution at centre point of side-wall

Pre-Heating Period: It is possible that, during the transition of flow from laminar to turbulent, existence of turbulent wakes and the formation of vortex pairs, may cause an intensive peak in rate of momentum and heat fluxes adjacent to walls.

Cycle Region: It is predicted that due to the simultaneous changes in wall temperature and ambient temperature (increasing ambient temperature and decreasing wall temperature) magnitude of momentum flux adjacent to wall changes too. As a result of this fact, instant change in the inertial term of momentum equation causes an increase in the fluctuation of instant velocity values. In other words, turbulence kinetic energy rapidly increases. In order to understand the mechanics of these peaks, two separated numerical studies are conducted for the cycle and pre-heating regions. Transient boundary conditions are acquired from the experiments conducted during these present and previous studies.

\subsection{Governing Equations}

A bousinesq-model (k-epsilon) is applied for the modeling of turbulence. Ideal gas assumption has been used for density variations.

Continuity equation:

$$
\frac{\partial \rho}{\partial t}+\frac{\partial\left(\rho u_{i}\right)}{\partial x_{i}}=0
$$

Momentum equation:

$$
\rho \frac{D u_{i}}{D t}=\frac{\partial p}{\partial x_{i}}+\frac{\partial}{\partial x_{j}}\left[\mu\left(\frac{\partial u_{i}}{\partial x_{j}}+\frac{\partial u_{j}}{\partial x_{i}}\right)-\rho \overline{u_{\imath}^{\prime} u_{\jmath}^{\prime}}\right]+\rho g_{i}
$$

Energy equation:

$$
\begin{aligned}
\rho C_{p} \frac{D T}{D t}=k \frac{\partial^{2} T}{\partial x_{i}^{2}} & +\mu \frac{\partial u_{i}}{\partial x_{j}}\left(\frac{\partial u_{i}}{\partial x_{j}}+\frac{\partial u_{j}}{\partial x_{i}}\right)+\frac{\partial}{\partial x_{i}}\left(-C_{p} \rho \overline{u_{\imath}^{\prime} T^{\prime}}\right) \\
& +S_{\text {energy }}
\end{aligned}
$$

Transport Equation - k:

$$
\begin{gathered}
\frac{\partial}{\partial t}(\rho k)+\frac{\partial}{\partial x_{i}}=\frac{\partial}{\partial x_{j}}\left[\left(\mu+\frac{\mu_{t}}{\sigma_{k}}\right) \frac{\partial k}{\partial x_{j}}\right]+P_{k}+P_{b}-\rho \epsilon-Y_{M} \\
+S_{k}
\end{gathered}
$$

Transport Equation $-\varepsilon$ :

$$
\begin{gathered}
\frac{\partial}{\partial t}(\rho \epsilon)+\frac{\partial}{\partial x_{i}}=\frac{\partial}{\partial x_{j}}\left[\left(\mu+\frac{\mu_{t}}{\sigma_{k}}\right) \frac{\partial \epsilon}{\partial x_{j}}\right]+C_{1 \epsilon} \frac{\epsilon}{k}\left(P_{k}+C_{3 \epsilon} P_{b}\right) \\
-C_{2 \epsilon} \rho \frac{\epsilon^{2}}{k}+S_{\epsilon}
\end{gathered}
$$

\subsection{Discretization of PDE's}

Pressure-Based Explicit approach has been applied to the problem. All transport equations are solved by second order schemes in order to prevent a possible numerical diffusion problem.

Body force weighted approach has been used for pressure, as the buoyancy forces dominances the flow regime.

\subsection{Stability and Consistency}

Time step size is chosen under the consideration of CFL condition. It is also provided that time step size is lower than the advective time scale.

\subsection{Numerical Results for Pre-Heating Region}

Numerical results are validated by the experimental data. Maximum numerical error comes out to be approximately $3 \%$. 
Due to the unstability of oven, it is possible to have experimental results having approximately $1 \% \sim 1.5 \%$, this is why present numerical results are accepted to be valid which the deviation of numerical results from experimental data shown in figure 14 .

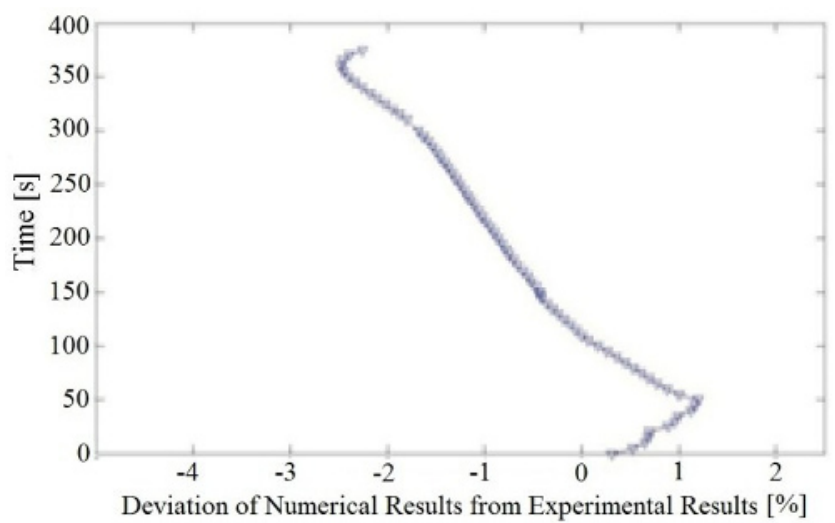

Fig. 14. Validation of Numerical Results by Experimental Results

Transient turbulence kinetic energy distribution in comparison to transient distribution of a dimensionless parameter, $\beta \Delta T$, which can be intoduced as a measure of buoyancy forces is given below.

As can be seen from figure 15 and 16 , due to the simultaneous change of boundary conditions, in other words, change of wall temperatures, transition to laminar and laminar to transition or turbulent regimes may exist.

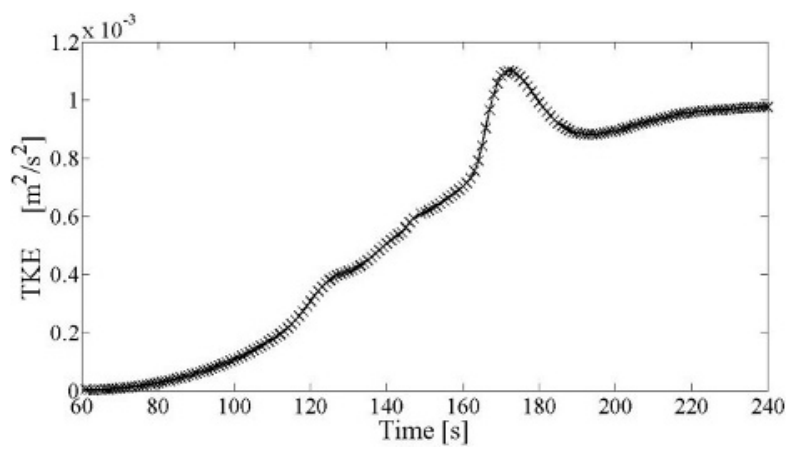

Fig. 15. Transient Turbulent Kinetic Energy Distribution at the adjacent cell of centre point of side-wall

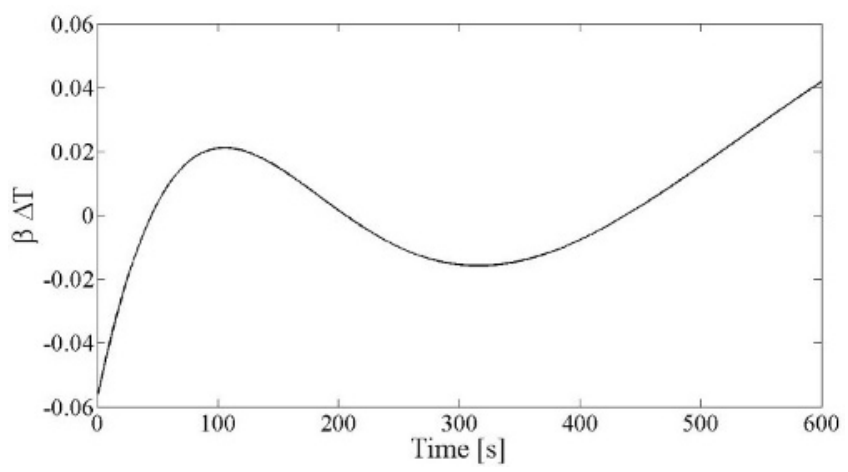

Fig. 16. Rayleigh Number for each time-step

This fact increases the possibility of prediction which states that turbulent kinetic energy peak mainly caused by deformation of vortex pairs.

In order to understand the mechanics of this instability, an approximate analysis has been carried out by the means of budget of turbulence kinetic energy. Budget of TKE* is given below,

$$
\frac{\partial k}{\partial t}=-u_{i} \frac{\partial k}{\partial x_{i}}+\frac{g}{\theta_{v}}\left(\overline{u_{g}^{\prime} \theta_{v}}\right)-\epsilon+\overline{u_{\imath}^{\prime} u_{\jmath}^{\prime}} \frac{\partial u_{i}}{\partial x_{j}}+\frac{\partial\left(\overline{\left.k u_{\imath^{\prime}}\right)}\right.}{\partial x_{i}}
$$

Respectively these terms resembles:

Local Storage/Tendency Term $=$ Advection of TKE by Mean Flow + Buoyancy Consumption Term - Viscous Dissipation + Shear Production/Loss Term + Turbulent Transport Term 


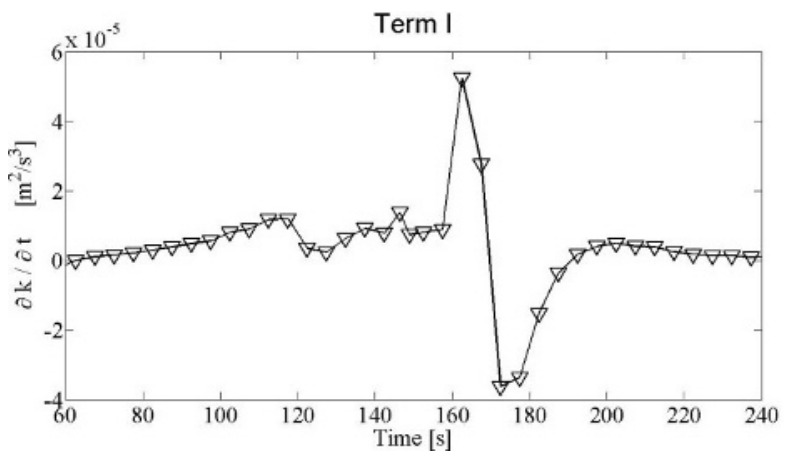

(a)

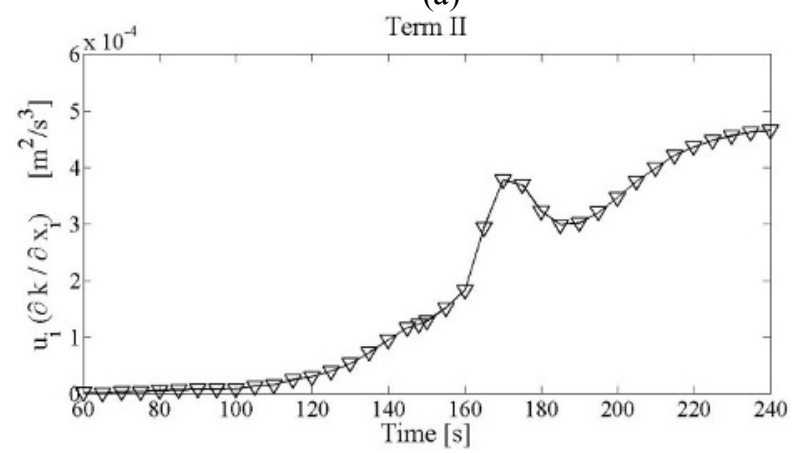

(b)

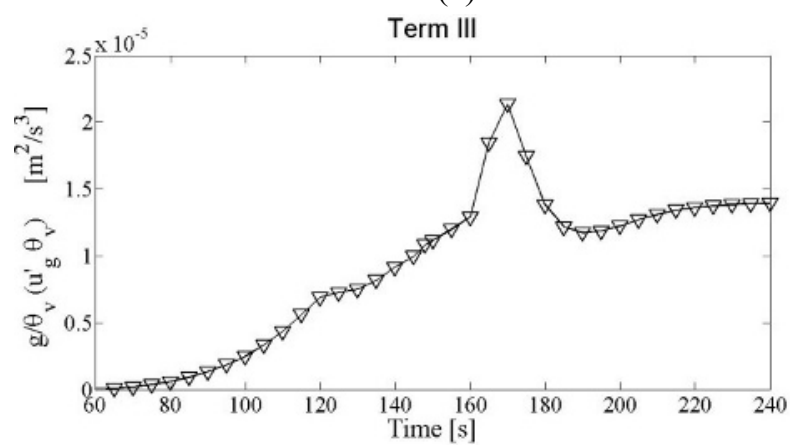

(c)

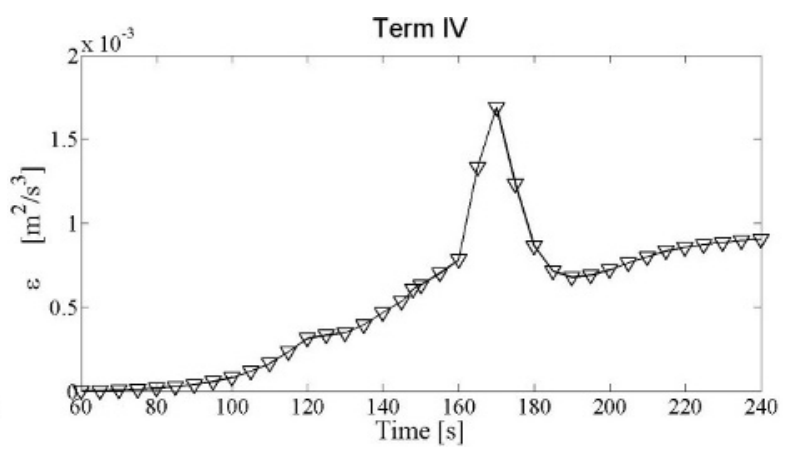

(d)

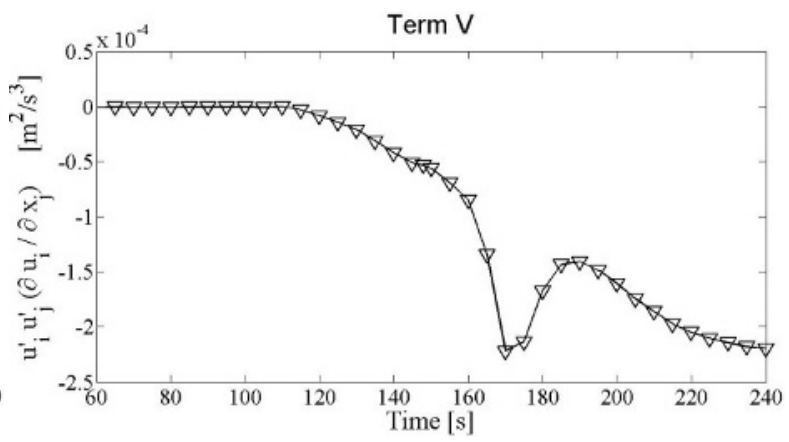

(e)

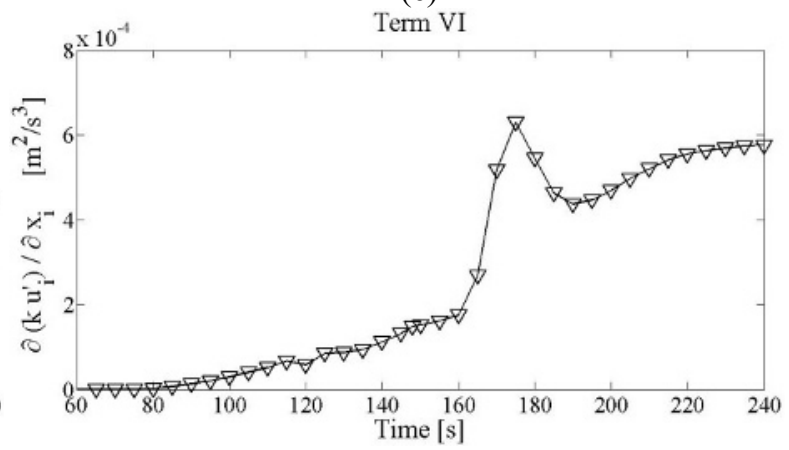

(f)

Fig. 17. Budget of Turbulent Kinetic Energy Graphs, (a : Local Storage Term, $b:$ Shear Production Term, $c:$ Buoyancy Production Term, $d$ : Viscous Dissipation Term, e : Turbulent Transport Term by Eddies, $f$ : Advection of TKE by mean flow)

As can be seen from figure 17-a, viscous dissipation takes more important role during the peak in comparison to the rest of time period. As wellknown, viscous dissipation occurs if $y^{+}<5$ (viscous sub-layer), since the position and kinematic viscosity is constant, it is likely to claim that change in $y^{+}$ depends on the change of $u_{\tau}$. So it can be stated, that frictional velocity decreased during the peak, in other way of expressing, dominance of viscous forces increased. As a result of this, vortex pairs and quasiturbulent wakes which are generated before the peak are dissipated by the viscous stresses. As the elements of total stress tensor increases with the formation of vortex pairs, by their dissipation, shear term in TKE becomes negative. It is also interesting that after dissipation process of energy cascade, the term of advection and turbulent transport by eddies increased, see figure 17-e and 17-f. This may also be explained by the fact that the instantaneous energy cascade process may cause further instabilities at a region closer to the referred point, as the rest of TKE is transported by remaining eddies through all directions. When the energy cascade is about to be completed, flow becomes to be less intented to be turbulent, see figure 17-a. Finally, it is likely to relate convective heat transfer coefficient peaks with buoyancy production and viscous dissipation. As expressed upwards, TKE is dissipated by viscous stresses as heat, in addition to this, as can been seen from figure 17-c, buoyancy is produced during this energy cascade process. In other words, buoyant forces become more dominant against viscous forces 
and this is how convective heat transfer coefficient rapidly increases in a similar fashion to figure 17-c and figure 17-d.

\section{Conclusion}

In the light of the initial energy budget study, the heat transfer mechanism of the original oven has been investigated and then two design proposals are trialed. According to the experimental results for the best case up to $6.7 \%$ of enhancement in energy consumption and $9.2 \%$ enhancement for baking time has been achieved.

It is found that, providing a diffusive dominated flow regime close to walls and advection dominated flow in the center of the cavity, leads to a better baking performance of the domestic oven,

As indicated before, experimentally it is found that convective heat transfer coefficient have remarkable peaks during baking process. According to the CFD study that has been conducted to understand the reasons underlying these peaks, rapid changes in the terms of budget of turbulent kinetic energy is the main reason.

\section{Acknowledgement}

The authors would like to thank The Scientific and Technological Research Council of Turkey (TUBITAK) and Arcelik R\&D Department personnel and managers for their valuable contributions and financial support.

\section{References}

1. C.Scarisbrick, M. Newbrough, S.D. Probert, Applied Energy, 39, (1991)

2. European Committee for Electrotechnical Standardization (CELENEC), 2001. Electric ovens for household use - Methods for measuring energy consumption, EN 50304 (2001)

3. Hipor 450 porous test brick, http://www.skamol.com/media/hipor_450.pdf (internet access: 20.09.2012)

4. O. Isik, S. U. Onbasioglu, Energy budget study of 9657 ETI built-in oven and energy saving by radiation heat transfer, Technical Report. (2011)

5. O. Temel, O. Isik, S.U. Onbasioglu, HEFAT, 3, 1345-1350 (2012) 